\title{
Bronchial Thermoplasty in Severe Asthma: Best Practice Recommendations from an Expert Panel
}

\author{
Peter I. Bonta ${ }^{a}$ Pascal Chanez ${ }^{b}$ Jouke T. Annema ${ }^{a}$ Pallav L. Shah ${ }^{c-e}$ \\ Robert Niven ${ }^{f}$ \\ ${ }^{a}$ Department of Respiratory Medicine, Academic Medical Center, University of Amsterdam, Amsterdam, \\ The Netherlands; ${ }^{b}$ Aix-Marseille Université, Clinique des bronches, allergie et sommeil/APHM, Marseille C2VN \\ Center INSEM INRA UMR1062, Marseille, France; ' Department of Respiratory Medicine, Royal Brompton Hospital, \\ London, UK; ${ }^{\mathrm{d}}$ National Heart and Lung Institute, Imperial College London, London, UK; ${ }^{\mathrm{e}}$ Department of Respiratory \\ Medicine, Chelsea and Westminster Hospital, London, UK; ${ }^{\mathrm{f}} \mathrm{MAHSC}$, University of Manchester and Manchester \\ Foundation Trust, Manchester, UK
}

\section{Keywords}

Bronchial thermoplasty · Bronchoscopy · Asthma

\begin{abstract}
Bronchial thermoplasty (BT) is a bronchoscopic treatment for patients with severe asthma who remain symptomatic despite optimal medical therapy. In this "expert best practice" paper, the background and practical aspects of BT are highlighted. Randomized, controlled clinical trials have shown BT to be safe and effective in reducing severe exacerbations, improving quality of life, and decreasing emergency department visits. Five-year follow-up studies have provided evidence of the functional stability of BT-treated patients with persistence of a clinical benefit. The Global Initiative for Asthma (GINA) guidelines state that BT can be considered as a treatment option for adult asthma patients at step 5. Patient selection for BT requires close collaboration between interventional pulmonologists and severe asthma specialists. Key patient selection criteria for BT will be reviewed. BT therapy is delivered in 3 separate bronchoscopy sessions at least 3 weeks apart, covering different regions of the lung separately. Patients are treated with $50 \mathrm{mg} /$ day of predniso-
\end{abstract}

lone or equivalent for 5 days, starting treatment 3 days prior to the procedure. The procedure is performed under moderate-to-deep sedation or general anesthesia. At bronchoscopy a single-use catheter with a basket design is inserted through the instrument channel and the energy is delivered by a radiofrequency (RF) generator (Alair ${ }^{\mathrm{TM}}$ Bronchial Thermoplasty System). BT uses temperature-controlled RF energy to impact airway remodeling, including a reduction of excessive airway smooth muscle within the airway wall, which has been recognized as a predominant feature of asthma. The treatment should be performed in a systemic manner, starting at the most distal part of the (sub)segmental airway, then moving proximally to the main bronchi, ensuring that the majority of the airways are treated. In general, 40-70 RF activations are provided in the lower lobes, and between 50 and 100 activations in the upper lobes combined. The main periprocedural adverse events are exacerbation of asthma symptoms and increased cough and sputum production. Occasionally, atelectasis has been observed following the procedure. The long-term safety of BT is excellent. An optimized $B T$ responder profile - i.e., which specific asthma phenotype benefits most - is a topic of current research.

(C) 2018 The Author(s)

Published by S. Karger AG, Basel

Dr. Peter I. Bonta, MD, PhD

Department of Respiratory Medicine, Academic Medical Center Meibergdreef 9

NL-1105 AZ Amsterdam (The Netherlands)

E-Mail p.i.bonta@amc.uva.nl
This article is licensed unde the Creative Commons Ath NonComicte is licensed under the Creative Com NC-ND) (hial-NoDerivatives 4.0 International License (CC BYUsage and distribution for commercial purposes as well as any distribution of modified material requires written permission. 


\section{Introduction}

Bronchial thermoplasty (BT) is a bronchoscopic treatment option for patients with severe asthma that is based on local radiofrequency (RF) energy delivery to the larger airways [1]. RF energy is applied through the Alair ${ }^{\mathrm{TM}}$ Bronchial Thermoplasty System (Boston Scientific, Natick, MA, USA), which consists of a single-use basket catheter and an RF energy generator (Fig. 1). During bronchoscopy, RF energy "activations" are delivered using the basket catheter in airways $2 \mathrm{~mm}$ or larger, and each activation heats the exposed airway section to approximately $65^{\circ} \mathrm{C}$. Although the mechanism of action of BT is incompletely understood, there is evidence that BT mainly acts through intervening in airway remodeling by reducing airway smooth muscle (ASM) and potentially by modulating the composition of the extracellular matrix [2]. As such, BT is considered the first asthma treatment that targets airway remodeling instead of mainly modulating airway inflammation and bronchomotor tone. However, BT might modulate inflammation as well through the reduction of ASM, airway extracellular matrix, and/or airway innervation. Studies are ongoing to investigate the mechanism of action of BT and related biomarkers involved and to correlate these to clinical outcome.

This statement is written by experts in BT and severe asthma from various European countries. Besides having hands-on and clinical expertise, the authors are actively involved in research regarding this topic. This paper discusses the background, practical and effective approaches to patient selection, patient preparation, procedural tips and tricks, patient management, and postprocedural care and follow-up in BT.

\section{Clinical Efficacy of BT}

The clinical efficacy of BT was studied in three randomized controlled trials (RCTs) 2007-2010 (Table 1) and their 5-year long-term follow-up studies 2011-2013 (Table 2) [2]. The first RCT was the AIR trial, which was an unblinded trial in 112 patients with moderate-tosevere asthma [3]. Patients treated with BT showed a reduction in mild exacerbations as compared with baseline, whereas they were unchanged in the control group. Furthermore there was a significant improvement in asthma control (Asthma Control Questionnaire [ACQ]) and quality of life (Asthma Quality of Life Questionnaire [AQLQ]) in BT-treated patients as compared to controls.

The second RCT was the RISA trial [4]. In this unblinded trial, 32 severe asthma patients were analyzed for BT safety and efficacy and showed a significant improvement in quality of life (AQLQ), asthma control (ACQ), rescue medication use, and pre-bronchodilator $\mathrm{FEV}_{1}$ percent predicted in BT-treated subjects versus controls. These results persisted when oral corticosteroids (OCS)
Fig. 1. Alair ${ }^{\mathrm{TM}}$ Bronchial Thermoplasty System. a Radiofrequency energy generator with a foot switch (black) to start an activation and a ground patch (blue) to close the energy circle. A basket catheter is plugged in (gray), with the green light (left corner) switched on, indicating that the system is ready to use. b Bronchial thermoplasty catheter handle (green) with a deployable basket catheter. c Basket catheter in a closed position. $\mathbf{d}$ Basket catheter in a fully expanded, open position. The black stripes/markings on the catheter are $5 \mathrm{~mm}$ apart and indicate the distance the catheter has to be retracted before a next activation can be safely provided.
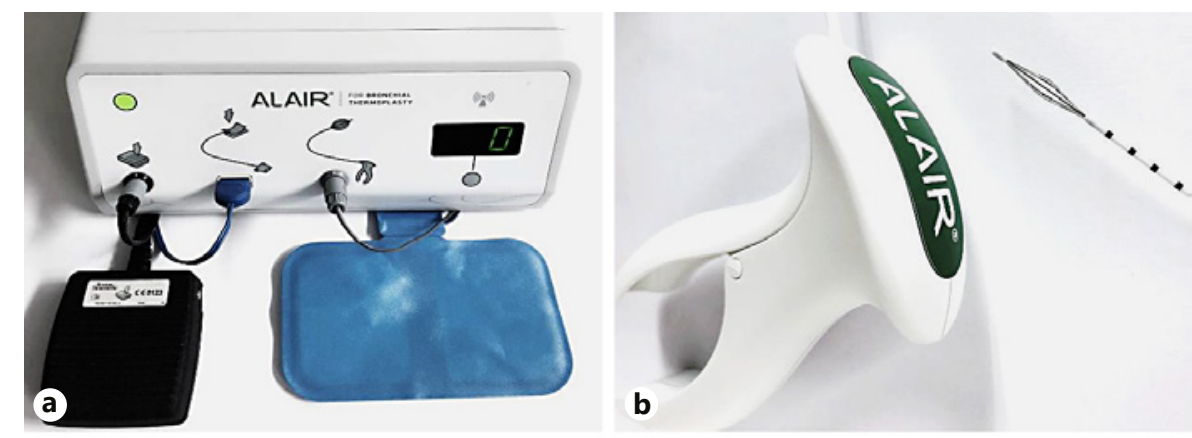

c

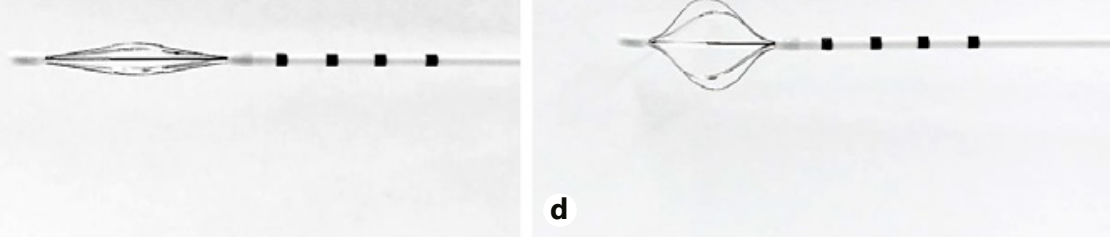


and inhaled corticosteroids (ICS) were reduced, except for the pre-bronchodilator $\mathrm{FEV}_{1}$ percentage predicted.

In the largest trial, the randomized double-blind sham-controlled AIR2 trial, 297 severe asthma patients were randomized 2:1 BT versus sham [5]. The primary endpoint demonstrated a clinically meaningful and significant improvement in AQLQ score of 0.5 or greater in the BT-treated patients, which to a lesser extent was also found in the sham-controlled group, most probably due to a placebo effect. A larger proportion of BT subjects than sham group subjects experienced a clinically meaningful within-subject improvement in AQLQ score of 0.5 or greater. Furthermore, the secondary endpoints demonstrated consistent benefits: fewer severe exacerbations, fewer emergency department visits, and fewer days missed from work were observed in the BT group.

For all three RCTs, 5-year long-term follow-up data are available, which are summarized in Table 2 [6-9]. Recently, a paper comparing the first 190 subjects from the FDA-mandated PAS2 registry with the 190 BT-treated

Table 1. Bronchial thermoplasty randomized controlled trials

\begin{tabular}{llllll}
\hline $\begin{array}{l}\text { Trial (design) } \\
\text { [Ref.], year }\end{array}$ & Patients, $n$ & $\begin{array}{l}\text { Follow-up, } \\
\text { months }\end{array}$ & $\begin{array}{l}\text { Pre-FEV } 1 \\
\text { baseline, } \% \text { pred. } \\
\text { (BT vs. control) }\end{array}$ & $\begin{array}{l}\text { Primary endpoint } \\
\text { (BT vs. control) }\end{array}$ & $\begin{array}{l}\text { Main (secondary) endpoints } \\
\text { (BT vs. control) }\end{array}$ \\
\hline $\begin{array}{l}\text { AIR (RCT) } \\
{[3], 2007}\end{array}$ & 112 & 12 & 72.7 vs. 76.1 & $\begin{array}{l}\text { Improvement in mild } \\
\text { exacerbation rate (per } \\
\text { patient/week) }-0.16 \text { vs. } \\
0.04(p=0.005)\end{array}$ & $\begin{array}{l}\text { Improvement in AQLQ, ACQ, } \\
\text { morning peak expiratory flow, } \\
\text { asthma symptom-free days, and } \\
\text { symptom scores }\end{array}$ \\
\hline $\begin{array}{l}\text { RISA (RCT) } \\
{[4], 2007}\end{array}$ & 32 & 52 & 62.9 vs. 66.4 & $\begin{array}{l}\text { Safety: short-term increase } \\
\text { in asthma-related morbidity; } \\
\text { long-term improvement }\end{array}$ & $\begin{array}{l}\text { Improvement in AQLQ, ACQ, and } \\
\text { Reduction in rescue medication use }\end{array}$ \\
\hline $\begin{array}{l}\text { AIR2 (RCT, } \\
\text { sham controlled) } \\
{[5], 2010}\end{array}$ & 297 & 12 & & $\begin{array}{l}\text { Improvement in AQLQ } \\
(1.35 \text { vs. 1.16) (PPS 0.96) }\end{array}$ & $\begin{array}{l}\text { Reduction in severe exacerbations, } \\
\text { emergency department visits, and } \\
\text { days missed from work/school }\end{array}$
\end{tabular}

BT, bronchial thermoplasty; RCT, randomized controlled trial; $\mathrm{FEV}_{1} \%$ pred., forced expiratory volume in $1 \mathrm{~s}$ percent predicted; ACQ, Asthma Control Questionnaire; AQLQ, Asthma Quality of Life Questionnaire; PPS, posterior probability of superiority.

Table 2. BT 5-year long-term follow-up studies and real-world registration

\begin{tabular}{|c|c|c|c|}
\hline Trial (design) [Ref.] & $\begin{array}{l}\text { Patients } \\
\text { (BT treated), } n\end{array}$ & $\begin{array}{l}\text { Follow-up, } \\
\text { years }\end{array}$ & Main outcomes \\
\hline AIR (extension study) [8] & 45 & 5 & Stable $\mathrm{FEV}_{1}$ and long-term safety profile \\
\hline RISA (extension study) [7] & 14 & 5 & $\begin{array}{l}\text { Stable } \mathrm{FEV}_{1} \text {, reduction in hospitalizations } \\
\text { and emergency department visits }\end{array}$ \\
\hline AIR2 (extension study) [40] & 162 & 5 & $\begin{array}{l}\text { Stable } \mathrm{FEV}_{1} \text { and long-term safety profile } \\
\text { including chest HRCT } \\
44 \% \text { decrease in exacerbations } \\
78 \% \text { decrease in emergency department visits }\end{array}$ \\
\hline $\begin{array}{l}\text { PAS } 2 \text { (post-FDA-approval } \\
\text { study) [6] }\end{array}$ & 190 & 3 & $\begin{array}{l}\text { Stable } \mathrm{FEV}_{1} \\
45 \% \text { decrease in severe exacerbations } \\
55 \% \text { decrease in emergency department visits } \\
40 \% \text { decrease in hospitalizations }\end{array}$ \\
\hline
\end{tabular}

BT, bronchial thermoplasty; $\mathrm{FEV}_{1}$, forced expiratory volume in $1 \mathrm{~s}$; HRCT, high-resolution computed tomography. 
subjects from the AIR2 trial at 3 years of follow-up (matched sample sizes) was published [6]. The percentage of PAS2 asthma patients treated with BT that experienced severe exacerbations, emergency department visits, and hospitalizations significantly decreased by 45,55 , and $40 \%$, respectively, echoing the AIR2 results. Similar results were observed for severe exacerbations, emergency department visits, and hospitalization rates (events per patient per year). Moreover, the percentage of PAS2 patients after BT using systemic OCS decreased by $46 \%$; however, the OCS dose was higher in those patients that remained on OCS.

In the last decade, globally several cohorts of severe asthma patients treated with BT have been reported with positive results in clinical outcome parameters and a favorable safety profile [10-18]. Unfortunately, so far none of these studies were able to define a specific responder profile.

\section{Key Messages}

- In RCTs and (large) cohort studies, BT has been shown to be effective in reducing severe exacerbations, improving quality of life, and decreasing emergency department visits in severe asthma patients.

- Five-year long-term follow-up studies have shown BT to be safe (stable pulmonary function test and no bronchiectasis on chest $\mathrm{CT}$ ) with persistent reductions in asthma exacerbation rates and/or emergency department visits/hospitalizations.

- The real-world PAS2 registry mirrors the results seen in the RCTs for BT regarding severe exacerbations, emergency department visits, and hospitalizations.

\section{Patient Selection}

Severe asthma is defined as asthma requiring the use of high-dose ICS next to a second controller and/or systemic OCS to prevent it from becoming uncontrolled or asthma that is uncontrolled despite this therapy $[19,20]$. As such, all patients that are selected for BT treatment should fulfil the ATS/ERS criteria for severe asthma. This group represents $3.6-10 \%$ of asthma patients and is known to have a high burden of disease with frequent asthma exacerbations and/or progressive lung function decline, resulting in excessive utilization of health care resources [21-24].

The treatment approach for severe asthma is described in the Global Initiative for Asthma (GINA) guideline steps 4 and 5. GINA step 4 consists of treatment with medium- to high-dose ICS with a LABA (long-acting $\beta$-adrenoceptor agonist), and/or an extra controller such as tiotropium, a leukotriene modifier, or theophylline. When asthma control is not achieved within GINA step 4, GINA step 5 advises add-on treatment including LAMA (long-acting muscarinic antagonists) - for instance, tiotropium and biologics (e.g., antiIgE and anti-interleukin-5 (anti-IL-5]) - and low-dose OCS. ${ }^{1}$ Driven by improved phenotyping, these add-on treatments are now increasingly prescribed to patients with distinct asthma phenotypes [20, 25, 26]. For example, anti-IgE can be considered for patients with a predominant allergic phenotype, and anti-IL-5 for patients with a predominant eosinophilic phenotype. For the noneosinophilic, nonallergic predominant phenotype, treatment with macrolides may be considered. BT may be considered for patients with severe asthma with predominant chronic airflow obstruction and patients with an unsatisfactory response to anti-IgE, anti-IL-5, or macrolides. ${ }^{2}$ Whether certain inflammatory phenotypes or responsiveness to specific asthma medication use correlates to the presence of irreversible airway obstruction and/or BT response is not clear and needs further investigation.

BT has been applied to patients with moderate-tosevere asthma, but it is currently mainly used for patients with severe asthma refractory to the available optimal medical maintenance therapy, including biologics. The key selection criteria are provided in Table 3 . The selection of candidate patients for BT should be rigorous and follow a step-by-step assessment from difficult to severe asthma. First of all, the diagnosis of asthma should be confirmed and should be based on the history of variable and reversible respiratory asthma symptoms with evidence of (reversible) airway obstruction by pulmonary function tests. Second, despite optimal maintenance asthma therapy including possible biologics, potential BT candidates' asthma remains uncontrolled; they have daily symptoms as measured by asthma control test (ACT) and/or ACQ and recurrent/frequent exacerbations, which results in a major burden on their quality of life as measured by an asthma-related quality-of-life questionnaire (e.g., AQLQ). Furthermore, adherence to asthma therapy should be checked, including the inhalation tech-

1 Global Initiative for Asthma: Global Strategy for Asthma Management
and Prevention, 2016 (www.ginasthma.org).
2 British Thoracic Society, Scottish Intercollegiate Guidelines Network:
British guideline on the management of asthma, 2016 (www.brit-thoracic.
org.uk_document-library_clinical-information_asthma_btssign-asthma-
guideline-2016); Global Initiative for Asthma: Global Strategy for Asthma
Management and Prevention, 2016 (www.ginasthma.org); Trivedi et al. [26].

Bonta/Chanez/Annema/Shah/Niven 
Table 3. Inclusion and exclusion criteria for BT

Main inclusion criteria

- Diagnosis of severe asthma as defined by the ATS/ERS criteria

- Age 18-65 years (age 65-75 years may be acceptable in experienced centers ${ }^{2}$ )

- Written informed consent for BT

- Willing and able to comply with the 3 bronchoscopy procedures

- High-dose ICS/LABA

- OCS $\leq 10 \mathrm{mg} /$ day (OCS $>10$ but $<40 \mathrm{mg} /$ day may be acceptable in experienced BT centers ${ }^{2}$ )

- May also be taking leukotriene modifiers and/or anti-IgE

- Pre-bronchodilator $\mathrm{FEV}_{1} \%$ pred. $\geq 60 \%$ (pre-bronchodilator $\mathrm{FEV}_{1} \%$ pred. $>30$ but $<60 \%$ may be acceptable in experienced BT centers $^{2}$ )

- ACQ score $>1.5$

- AQLQ score $\leq 6.25$

- Nonsmoker for $\geq 1$ year (if former smoker: $<10$ pack-years total smoking history)

Main exclusion criteria ${ }^{1}$

- Having an asthma exacerbation

- History of life-threatening asthma (previous intubation or ICU admission in the prior 2 years)

$-\geq 4$ lower respiratory tract infections in the previous 12 months

$-\geq 3$ hospitalizations for asthma in the previous 12 months

- Known sensitivity to medications required to perform bronchoscopy

- $\mathrm{BMI}>35$

- Other respiratory diseases including emphysema, cystic fibrosis, vocal cord dysfunction, mechanical upper airway obstruction, eosinophilic granulomatosis with polyangiitis (EGPA), or allergic aspergillosis

- Segmental atelectasis, lobar consolidation, significant or unstable pulmonary infiltrate, or pneumothorax confirmed by chest radiography

- Cardiovascular disease including myocardial infarction, angina, cardiac dysfunction, cardiac dysrhythmia, conduction defect, cardiomyopathy, or stroke

- Known aortic aneurysm

- Significant comorbid illness including cancer, renal failure, liver disease, or cerebrovascular disease

- Uncontrolled hypertension

- Implanted electrical stimulation device

- Known coagulopathy and/or platelet disorders

- Pregnancy

- Any other medical condition that could interfere with the BT procedure

BT, bronchial thermoplasty; ICS, inhaled corticosteroids; LABA, long-acting $\beta$-adrenoceptor agonists; OCS, oral corticosteroids; $\mathrm{FEV}_{1} \%$ pred., forced expiratory volume in 1 s percent predicted; ACQ, Asthma Control Questionnaire; AQLQ, Asthma Quality of Life Questionnaire; BMI, body mass index. ${ }^{1}$ Bronchial Thermoplasty in Severe Persistent Asthma (PAS2); NCT01350336. ${ }^{2}$ Bicentric Prospective Study, Evaluating Bronchial THERMOPLASTY in a Patient Presenting Severe Uncontrolled Asthma (ASMATHERM); NCT01777360.

nique of the patient. During an initial longitudinal assessment, the environment including potential asthmaaggravating factors (e.g., allergens, in- and outdoor pollution, and occupational exposures) as well as comorbid conditions have to be investigated and treated, including psychological management. Further relevant comorbidities should be excluded and/or treated. For this purpose, clinical, laboratory (including autoimmune serology testing), functional, and imaging assessments are instrumental and should include chest CT, cardiac screening, and assessment of vocal cord dysfunction.

\section{Lung Function and Medication}

In the clinical BT program, $\mathrm{FEV}_{1}$ had been considered for patient eligibility, and in the different trials, different levels of airway obstruction as measured by $\mathrm{FEV}_{1}$ were used for the inclusion and exclusion criteria. Furthermore, asthma patients with irreversible airway obstruction, as reflected in lower levels of $\mathrm{FEV}_{1}$, have shown higher levels of ASM as a marker of clinically important airway remodeling [27]. Although not proven, there seems to be a positive correlation between severity of airway obstruction and clinical response after BT $[4,6,15]$. This is exemplified by the results of the RISA trial, in 
which patients with a lower $\mathrm{FEV}_{1}$ were $\mathrm{BT}$ treated, as compared to the pivotal AIR2 trial. Furthermore, the recently published results of a nonrandomized French study showed that patients with an $\mathrm{FEV}_{1}$ as low as $30 \%$ with high exacerbation rates were BT treated with a favorable clinical response and acceptable safety profile [15, 16]. An increased postprocedural incidence of hospitalizations following BT among subjects whose asthma is very severe was observed. Based on these data, the authors are of the opinion that patients with an $\mathrm{FEV}_{1}$ of $60-80 \%$ are preferred candidates for $\mathrm{BT}$, and that patients with an $\mathrm{FEV}_{1}$ of $30-60 \%$ can be BT treated in highly experienced BT centers. There is no change in lung function in patients BT treated either in clinical studies or in real-life clinical practice. In the clinical BT program, patients were eligible if treated with high-dose ICS (daily dose $>1,000$ $\mu \mathrm{g}$ beclomethasone or equivalent) and high-dose longacting $\beta$-adrenoceptor agonist $(>80 \mu \mathrm{g}$ salmeterol or equivalent). OCS were allowed at a maximum of $30 \mathrm{mg} /$ day, and biological therapy including omalizumab was permitted. Patients with a high frequency of rescue medication use ( $>8$ puffs of short-acting $\beta_{2}$-adrenergic agonist), potentially reflecting ongoing exacerbation/severe asthma instability, were excluded from the trials.

\section{Airway Hyperresponsiveness}

Bronchial hyperresponsiveness is a hallmark of asth$\mathrm{ma}$, and whenever it is possible to safely perform a methacholine or histamine challenge test, its result contributes to the diagnosis of asthma, especially where reversibility has not been clearly demonstrated. In the clinical BT program, patients were selected at least in part on the evidence of bronchial hyperresponsiveness expressed as a significant response to $\beta_{2}$-adrenergic agonists, deterioration on withdrawal from $\beta_{2}$-adrenergic agonists, or increased responsiveness to methacholine. However, except for the single-center feasibility trial in mild asthma patients, none of the randomized trials were able to show improvement in bronchial hyperresponsiveness to methacholine $\mathrm{PC}_{20}$ [1]. In the "real world," measuring bronchial hyperresponsiveness using a challenge test prior to BT may be problematic in severe asthma, especially when baseline respiratory function is already compromised. The last reports on real-world severe asthma, including the PAS2 registry, did not include any methacholine $\mathrm{PC}_{20}$ measures [6]. Chronic intractable cough has been reported to be improved by BT in severe asthma, even though the exact mechanism has not been investigated yet in a clinical setting (personal communication).

\section{Asthma Control and Exacerbations}

Asthma control can be assessed in various ways including asthma exacerbation rates and composite questionnaires of daily symptoms such as the ACT and ACQ. The effect of BT of a reduction in exacerbations was demonstrated in most of the studies of the clinical BT program and persisted during the posttreatment period up to 5 years; however, it may not be essential to only have a history of severe exacerbations to benefit from BT. In the real-world program, the decrease in exacerbations was around $50 \%$ overall and persisted, indicating a prolonged and sustained effect.

\section{Contraindications}

Obviously, patients experiencing an ongoing exacerbation should not be treated with BT. In the clinical BT program, patients were stable for at least 14 days prior to BT. Patients with ongoing or recurrent respiratory infections and/or (colonized) bronchiectasis are no good candidates for BT. Indeed, in general, patients with respiratory diseases other than asthma should not be treated with BT. This includes, but is not limited to, emphysema, cystic fibrosis, vocal cord dysfunction, untreated mechanical upper airway obstruction, eosinophilic granulomatosis with polyangiitis (EGPA), allergic aspergillosis, interstitial lung diseases, segmental atelectasis, lobar consolidation, significant or unstable pulmonary infiltrate, or pneumothorax confirmed by chest radiography. Furthermore, patients with a recent history of ICU admission with tracheal intubation should be considered as patients at high risk of post-BT complications, but there are no hard data that confirm this. Asthmatics with uncontrolled comorbidities such as, but not restricted to, cardiovascular and severe renal/liver disorders should not to be treated with BT. BT can be performed on patients on aspirin, but newer antiplatelet agents (e.g., clopidogrel), vitamin $\mathrm{K}$ antagonists (e.g., warfarin), and anticoagulation drugs (e.g., rivaroxaban) should be omitted during BT. Other well-known contraindications to BT are pacemakers/implantable cardiac defibrillators and intolerance to medications used during or in preparation of BT.

The obvious complexity and uncertainties in the selection of patients for BT described above require a multidisciplinary team approach including severe asthma specialists and interventional pulmonologists to ensure an optimal selection of patients for BT at centers that see high volumes of severe asthma patients and that have the complete panel of severe asthma treatment modalities including biologics at hand. 


\section{Key Messages}

- BT may be considered for adult patients with severe asthma that is poorly controlled despite optimal medical therapy.

- The complexity and uncertainties in the selection of patients for BT require a multidisciplinary team approach at asthma centers with high volumes of severe asthma patients and a high level of experience in interventional pulmonology procedures.

\section{Bronchial Thermoplasty}

\section{Patient Preparation and Management}

Patients planned for BT should be in an optimal stable condition, i.e., without any asthma exacerbation or respiratory infection for at least 14 days. In addition to the patients' standard medical therapy for their severe asthma, they should be pretreated with prednisolone at $50 \mathrm{mg} /$ day 3 days before BT, on the day of BT, and the day after BT. Vital sign assessments including pulse oximetry, a standard physical examination including breathing sounds, and $\mathrm{FEV}_{1}$ should be performed before the procedure. If the post-bronchodilator $\mathrm{FEV}_{1}$ is $<80 \%$ of a subject's baseline value, postponing the $\mathrm{BT}$ procedure should be considered. Before the procedure, all patients should be pretreated with nebulized salbutamol and/or ipratropium bromide.

\section{General Anesthesia and Sedation}

In the RCTs, both general anesthesia and sedation including propofol, midazolam, and fentanyl were allowed, and currently there are no data available for comparison. Therefore, in practice, the strategy chosen will largely depend on local expertise and availability. It is critically important to achieve and maintain the right level of anesthesia and sedation during the complete procedure, which in general lasts 30-60 min (a median of $44 \mathrm{~min}$ for the right and left lower lobes; a median of $58 \mathrm{~min}$ for both upper lobes) [28]. The experience of the authors is that during BT most severe asthma patients experience cough, dyspnea, and some level of pain. The general purpose of sedation and general anesthesia during BT is therefore to maintain optimal airway/breathing, cough, and pain control. Therefore, a form of moderate-to-deep sedation or general anesthesia is preferred over mild sedation with midazolam only. During the procedure, supplemental oxygen should be provided by a nasal cannula or by ventilation, during which an $\mathrm{FiO}_{2}<40 \%$ is recommended to avoid the theoretical possibility of airway ignition.

\section{General Anesthesia}

The advantage of general anesthesia is the ease of airway and patient management. The use of positive endexpiratory pressure and muscle relaxants allows the optimal control and secure positioning of the basket catheter needed to provide activations as precisely as possible. On the other hand, general anesthesia including intubation and ventilation could be seen as overtreatment, since this is associated with higher complication risks, including hemodynamic instability, and requires a more complex infrastructure incurring higher costs. During general anesthesia, minimal ventilator settings with low-frequency ventilator settings (8-10 times/min) and long expiration times (inspiration-to-expiration ratios of 1:3-4) are normally recommended.

\section{Sedation}

Several sedation strategies for BT have been reported, and the choice of strategy should follow guidelines according to country and institution. Several sedation strategies have been described, but the most commonly applied combinations are midazolam and fentanyl or propofol and fentanyl/remifentanil [28-30]. For the latter combination, a specialized sedation anesthesiology nurse-driven target-controlled infusion strategy has recently been successfully applied to BT, with favorable patient- and bronchoscopist-related outcomes [28]. An important consideration is that with an increased level of sedation, respiratory suppression and related carbon dioxide retention/hypoxemia can occur. Therefore, continuous monitoring during sedation is required, which includes, but is not limited to, respiration, the level of consciousness, pulse oximetry, and blood pressure. The advantage of both benzodiazepines like midazolam and opiates is that their effect can be readily reversed by flumazenil and naloxone, respectively. Sedation is further facilitated by the administration of topical lidocaine 1-4\% both to the airways and to the vocal cords/pharynx.

\section{Bronchoconstriction and Secretion Management}

Performing bronchoscopy on patients with severe asthma can be a challenge due to their unpredictable airway response, which might include bronchoconstriction as well as airway edema with mucus hypersecretion and mucosal bleeding. To avoid these responses, minimal touching of the airways by the bronchoscope and maximum avoidance of suction are recommended. The use of anticholinergic agents including atropine and glycopyrronium bromide to decrease secretion production may be considered. Also, increasing the dose of prednisolone to 


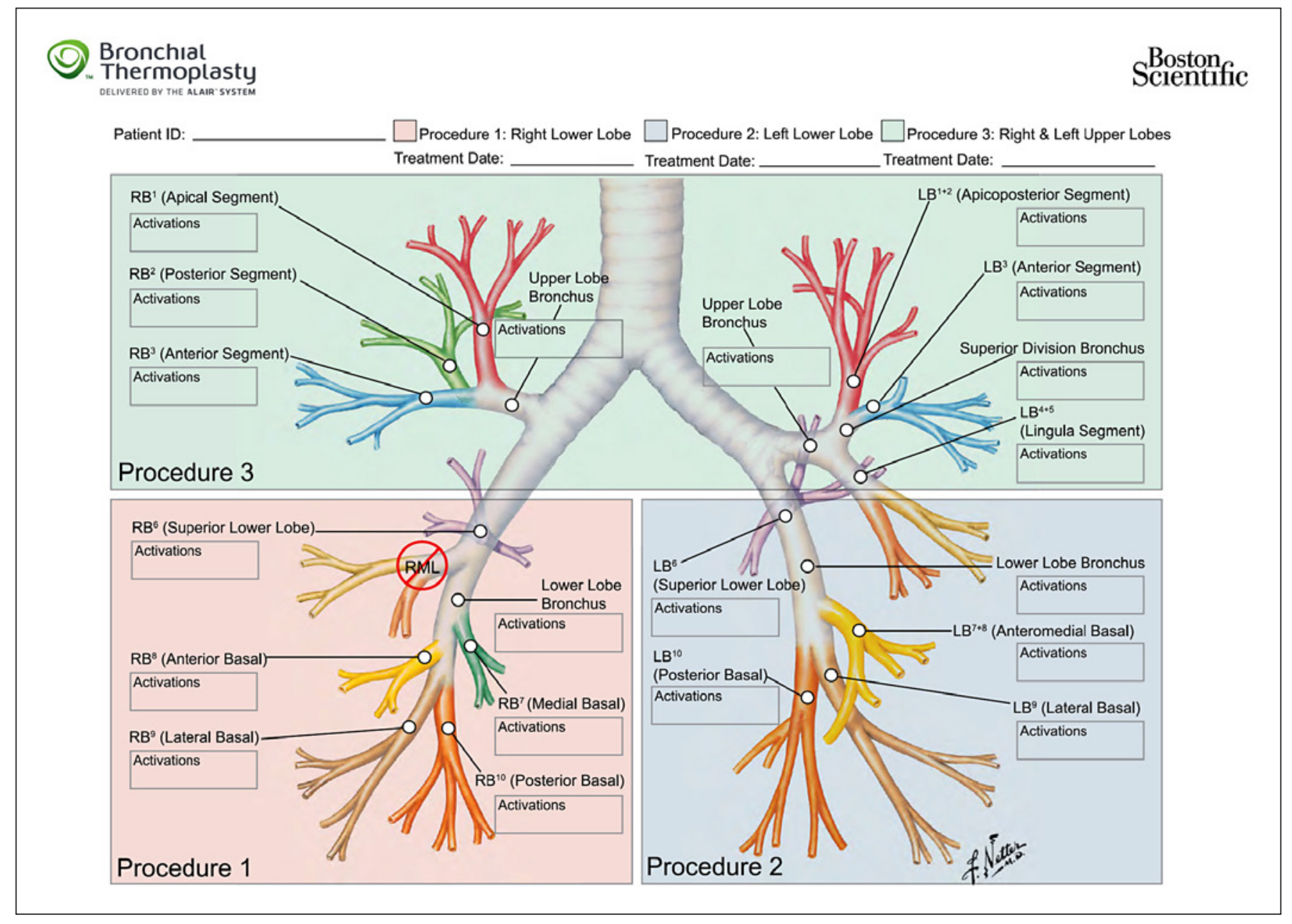

Fig. 2. Bronchial thermoplasty (BT) activation map. The map represents the bronchial tree and is divided into 3 procedures (procedures 1-3). Procedure 1: right lower lobe; procedure 2: left lower lobe; procedure 3: right and left upper lobes. Each white dot represents a BT activation site. During the procedures, the number of BT activations per activation site can be noted. No activations are provided in the right middle lobe (RML).

$1 \mathrm{mg} / \mathrm{kg}$ during the procedure might be considered for this purpose. To further diminish the bronchoconstrictive response, additional use of theophylline at a loading dose of $5 \mathrm{mg} / \mathrm{kg}$ followed by $9 \mathrm{mg} / \mathrm{kg} / 24 \mathrm{~h}$ continuous perfusion and 2,000 $\mathrm{mg}$ magnesium sulfate IV may be considered. The use of either of these agents should follow institutional guidelines, and physicians should exercise caution due to potential adverse effects.

\section{Bronchoscopy}

Inspection

Before BT is started, an inspection of the airways should be performed, with special attention to signs of tracheal/bronchial malacia, subglottic-tracheal airway stenosis, airway tumors, and other unexpected airway abnormalities. The authors recommend performing a bronchial wash for microbiological testing for the early recognition of (sub)clinical infections. If BT has been previously performed, the lobe that has been treated before should be carefully inspected for airway abnormalities, and if a mucosal abnormality is observed, it is advisable that the BT procedure be postponed and microbiological testing performed to exclude a secondary infection.

\section{Bronchial Thermoplasty}

A BT procedure is performed with 1 trained pulmonologist as the operator and 2 trained endoscopy nurses to handle the basket catheter and support the patient. A seda- 


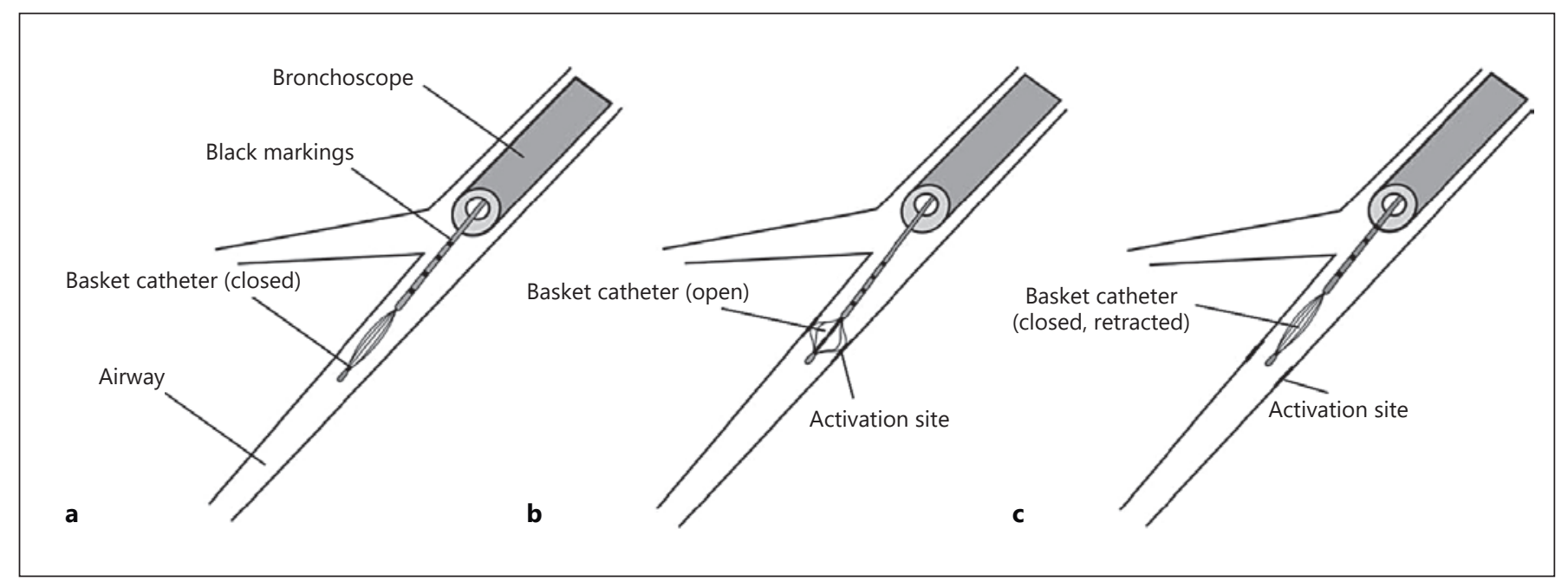

Fig. 3. Schematic representation of bronchial thermoplasty procedure. a During bronchoscopy, the bronchial thermoplasty basket catheter is advanced to a distal airway segment with the closed basket just in view. b Subsequently, the basket is gently opened by pressing the green handle to provide contact of the 4 struts with the airway wall. By pressing the foot switch, an activation is started for 10 s. c Directly thereafter, the basket catheter is closed again and retracted the distance of 1 black stripe/marking. By repeating this sequence $(\mathbf{a}-\mathbf{c})$ of basket catheter handling, all airways with a diameter between approximately 2 and $10 \mathrm{~mm}$ of a target lobe can be treated in a structured and systematic manner. tion specialist or anesthetist is preferable if possible. BT is provided with the Alair ${ }^{\mathrm{TM}}$ Bronchial Thermoplasty System (Boston Scientific), consisting of an RF controller connected to the patient by a return electrode to complete the electric circuit and a basket catheter that can be advanced through the instrument channel of a standard bronchoscope. Optimal BT treatment of all (sub)segmental airways may be more successful with next-generation, ultrathin, rotatable bronchoscopes with increased ease of use and higher degrees of tip flexibility (e.g., Olympus BF-P190).

During the initial inspection of the target lobe described above, the bronchial anatomy drives the BT treatment approach. The activations provided are recorded on a BT map (Fig. 2). In 3 sessions, the right lower lobe, the left lower lobe, and finally both upper lobes are treated typically 3-6 weeks apart. The right middle lobe is currently not treated, due to potential susceptibility of the right middle lobe to transient obstruction and hence atelectasis and right middle lobe syndrome.

In the lower lobes, BT is often initiated in the most distal subsegmental airways, commonly LB/RB 10. In the upper lobes, BT is often initiated in the apical subsegmental airways (LB/RB1). BT is performed by advancing the basket catheter through the instrument channel of the bronchoscope until 4 black stripes/markings $(2.0 \mathrm{~cm})$ are out and the struts of the basket catheter are just left in view to the distal targeted airway (Fig. 3a). Then the bas- ket catheter is opened and the activation is provided for about $10 \mathrm{~s}$ by pressing the foot switch (Fig. 3b, 4a). Subsequently, the catheter is closed and retracted $5 \mathrm{~mm}$ (represented by 1 black stripe/marking on the catheter), then opened again to deliver the next activation (Fig. 3c). This maneuver is repeated till the basket catheter has reached all (sub)segmental airways of that lobe, including the proximal large airways (Fig. 4b).

After each activation, mild blanching of the mucosa can occur (Fig. 4c). If one of the struts does not adequately oppose the airway wall, the generator will give an alarm and the activation will cease. Repeat closure and opening of the basket catheter is usually sufficient to overcome this problem. By repeating this maneuver, in general between 40 and 70 activations are provided in the lower lobes, and between 50 and 100 activations in the two upper lobes combined, depending on the patient's size and airway caliber.

It important to closely monitor which (sub)segments have been treated during the procedure, since accidental delivery of two activations to a single part of the airway is theoretically hazardous (e.g., risk of bleeding, bronchiectasis). On the other hand, accidentally omitting BT activations in some of the airways might hamper the full potential benefit of BT. Indeed, although this was not reported in the major clinical trials, recently the number of $\mathrm{BT}$ activations provided has been linked to BT outcome 

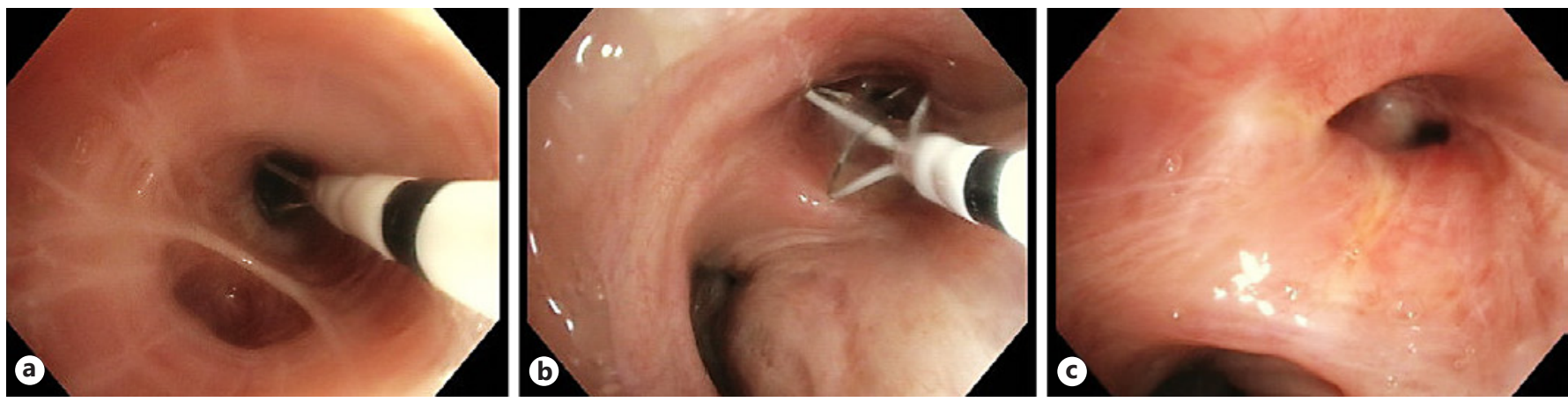

Fig. 4. Bronchoscopic pictures of a bronchial thermoplasty treatment of a right upper lobe. a Bronchial thermoplasty (BT) activation of a distal airway segment with the basket catheter in an open position with the struts just in view. $\mathbf{b}$ BT activation of a proximal airway (ostium of the right upper lobe) with the basket catheter fully expanded. c Blanching of the mucosa of the ostium of the right upper lobe directly after a BT activation.

[13]. Indeed, post-BT chest CT scans have shown that specific radiological patterns can be distinguished in airways directly exposed to RF energy, even though distant airway/lung areas not directly exposed can show abnormalities after BT as well [31-34].

Other than the aforementioned chest CT, there is currently no modality available that provides immediate feedback on the quality of a BT treatment. Optical coherence tomography imaging during bronchoscopy immediately after BT might qualify for this purpose, since this imaging modality has recently been shown to be able to image the airway wall at a near-histological level $[35,36]$. As the procedure requires advanced bronchoscopy skills, we recommend that only BT-trained (interventional) pulmonologists that regularly perform BT (e.g., $>10$ procedures a year) perform this procedure.

\section{Key Messages}

- BT should only be performed in a fully equipped bronchoscopy suite.

- Prior to performing the procedure, it has to be ensured that appropriate training, equipment, medications, and staff are in place to handle any potential bronchoscopic, respiratory, or anesthesia-related emergencies.

- BT can be performed under both (moderate-to-deep) sedation and general anesthesia as long as optimal cough/catheter control is achieved.

- A full BT treatment consists of 3 procedures, during which the right lower lobe, the left lower lobe, and both upper lobes are subsequently treated at least 3 weeks apart.

- BT should be performed in a systematic manner, starting at the most distal aspect and moving proximally to the bronchi, ensuring that the majority of the airways are treated.

\section{Follow-Up after BT}

\section{Short-Term Follow-Up and Acute Complication Management}

Patients are routinely kept for $4 \mathrm{~h}$ as a minimum, and overnight in some centers, as routine practice. As there are no comparisons of outcomes between a day case procedure and overnight admission, this decision should be based on local treatment protocols and experience. Measurement of posttreatment $\mathrm{FEV}_{1}$ or chest X-ray control is not routinely needed, although it was included in the original trials. The experience of the authors is that attempting spirometry can cause pain, coughing, and distress and does not assist the physician in discharge planning, as suboptimal recordings are likely to be obtained. If discharged, patients should have recourse to expert advice from a specialist center, and it is routine practice to contact patients by telephone as a minimum requirement after $24 \mathrm{~h}$ and 7 days.

Readmission is rarely necessary, but a number of short-term hospitalizations have been observed in the clinical trials and in the follow-up of patient cohorts. Worsening of asthma control after BT is to be expected - and unavoidable considering the impact of denuding the airway mucosa. The duration of asthma worsening is highly variable, but in general ranging from minimal to 1-2 weeks. Patients are counselled on this, and increased bronchodilator use, mucous clearance techniques, and extension of augmented OCS may all be required.

More serious side effects are the development of atelectasis or collapse of an airway by mucous plugging. Imaging by chest X-ray and/or chest CT can be of additional value to evaluate the extent of these side effects, al- 
though after each BT procedure radiological abnormalities are described to some degree in almost all patients [31, 34]. In rare cases, bronchoscopy is required to remove a plug or cast.

Posttreatment episodes of significant airway bleeding have been reported, which in one case report required bronchial artery embolization [37]. Furthermore, pulmonary infections requiring antibiotics have been mentioned, and for this reason the authors recommend a bronchial wash during BT bronchoscopy. One significant complication experienced and reported by one of the authors was a lung abscess that developed 1 week after a second treatment, occurring in the treated lobe [38]. The patient recovered completely with aggressive antibiotic therapy, but it resulted in a protracted hospital stay for the patient. Despite this, the patient elected to complete her treatment some months later. One case of pneumothorax and cyst formation has also been reported [39].

\section{Long-Term Follow-Up}

In the original treated trial population, no significant long-term side effects were observed. The reduction in exacerbations seen in the first year after treatment remained stable for up to 5 years [7-9]. Follow-up chest CT scans were performed on subpopulations of the treated patient cohort only, and there was no evidence of the development of bronchial strictures or bronchiectasis [9]. The results of additional real-world registries are expected, and hopefully some of these will include radiological and even pathological data.

The authors believe that all patients undergoing BT treatment in any setting (an ongoing research trial or as part of a national or international registry) should have a minimum of one annual follow-up assessment, and this should include pulmonary function testing; AQLQ, ACQ, and/or ACT administration; assessment of exacerbation frequency (including steroid bursts); assessment of health care resource utilization; medication burden; and measurement of inflammation including blood eosinophils, fractional exhaled nitric oxide, and sputum eosinophils if possible. We also believe that repeat ([ultra]low-dose) chest CT scanning should be considered at least every 5 years, and local protocols could be used to ensure this is not contrary to local ethical guidance.

\section{Should Patients Who Responded to BT Ever Be \\ Retreated?}

This is one of the frequently asked questions. It is clearly most relevant to patients who have been treated once with an initial good response but who then experi- ence deterioration in their control over a long time period. Some of the authors have patients followed up as long as 14 years from first treatment from the early trials, and whilst only rarely, this question has been asked by patients as well as health care professionals. There is currently a complete lack of evidence to support such action, and if ever it were to be considered, it should be part of a formalized research activity, which may need international collaboration, to collect meaningful data.

\section{Should the Middle Lobe Be Treated?}

Historically, the middle lobe was not treated, for fear of atelectasis and right middle lobe syndrome. Based on the currently available experience and evidence there seems little basis for not treating the middle lobe. We would welcome a clinical trial that includes middle lobe treatment with careful monitoring of its potential impact.

\section{Key Messages}

- BT may be performed as day care treatment with a minimum of $4 \mathrm{~h}$ of observation after treatment or include overnight admission based on local treatment protocols and experience.

- Patients undergoing BT treatment in any setting should be offered follow-up in the longer term to monitor clinical, imaging, and functional outcomes including potential adverse events.

\section{Conclusions}

BT for severe bronchial asthma is a treatment option for adult patients with uncontrolled asthma despite optimal therapy. These clinical best practice recommendations should aid physicians in patient selection, maximizing response rates and patient outcomes with BT treatment.

\section{Financial Disclosure and Conflicts of Interest}

The authors' views and opinions are personal. This best practice paper was initiated and executed by the authors independent of the manufacturer of the Alair ${ }^{\mathrm{TM}}$ Bronchial Thermoplasty System (Boston Scientific). P.I.B. and J.T.A. have received investigatorinitiated research grants from Boston Scientific and P.I.B. has received institutional fees for lectures from Boston Scientific, research grants from ZonMw (grant No. 90713477), and research grants from the Dutch Lung Foundation (grant No. 5.2.13.064JO). P.L.S. has received personal lecture fees from Boston Scientific. 


\section{References}

1 Cox G, Miller JD, McWilliams A, Fitzgerald JM, Lam S: Bronchial thermoplasty for asthma. Am J Respir Crit Care Med 2006;173: 965-969.

2 d'Hooghe JNS, Ten Hacken NHT, Weersink EJM, Sterk PJ, Annema JT, Bonta PI: Emerging understanding of the mechanism of action of Bronchial Thermoplasty in asthma. Pharmacol Ther 2018;181:101-107.

3 Cox G, Thomson NC, Rubin AS, Niven RM, Corris PA, Siersted HC, et al: Asthma control during the year after bronchial thermoplasty. N Engl J Med 2007;356:1327-1337.

4 Pavord ID, Cox G, Thomson NC, Rubin AS, Corris PA, Niven RM, et al: Safety and efficacy of bronchial thermoplasty in symptomatic, severe asthma. Am J Respir Crit Care Med 2007;176:1185-1191.

5 Castro M, Rubin AS, Laviolette M, Fiterman J, De Andrade Lima M, Shah PL, et al: Effectiveness and safety of bronchial thermoplasty in the treatment of severe asthma: a multicenter, randomized, double-blind, shamcontrolled clinical trial. Am J Respir Crit Care Med 2010;181:116-124.

6 Chupp G, Laviolette M, Cohn L, McEvoy C, Bansal S, Shifren A, et al: Long-term outcomes of bronchial thermoplasty in subjects with severe asthma: a comparison of 3-year follow-up results from two prospective multicentre studies. Eur Respir J 2017;50:1700017.

7 Pavord ID, Thomson NC, Niven RM, Corris PA, Chung KF, Cox G, et al: Safety of bronchial thermoplasty in patients with severe refractory asthma. Ann Allergy Asthma Immunol 2013;111:402-407.

8 Thomson NC, Rubin AS, Niven RM, Corris PA, Siersted HC, Olivenstein R, et al: Longterm (5 year) safety of bronchial thermoplasty: Asthma Intervention Research (AIR) trial. BMC Pulm Med 2011;11:8

9 Wechsler ME, Laviolette M, Rubin AS, Fiterman J, Lapa e Silva JR, Shah PL, et al: Bronchial thermoplasty: long-term safety and effectiveness in patients with severe persistent asthma. J Allergy Clin Immunol 2013;132: 1295-1302.

10 Arrigo R, Failla G, Scichilone N, La Sala A, Galeone C, Battaglia S, et al: How effective and safe is bronchial thermoplasty in "real life" asthmatics compared to those enrolled in randomized clinical trials? Biomed Res Int 2016;2016:9132198.

11 Chakir J, Haj-Salem I, Gras D, Joubert P, Beaudoin ĖL, Biardel S, et al: Effects of bronchial thermoplasty on airway smooth muscle and collagen deposition in asthma. Ann Am Thorac Soc 2015;12:1612-1618.

12 Iikura M, Hojo M, Nagano N, Sakamoto K, Kobayashi K, Yamamoto S, et al: Bronchial thermoplasty for severe uncontrolled asthma in Japan. Allergol Int 2017, Epub ahead of print.
13 Langton D, Sha J, Ing A, Fielding D, Thien F, Plummer V: Bronchial thermoplasty: activations predict response. Respir Res 2017;18: 134.

14 O'Reilly A, Browne I, Watchorn D, Egan JJ, Lane S: The efficacy and safety of bronchial thermoplasty in severe persistent asthma on extended follow-up. QJM 2018;111:155-159.

15 Pretolani M, Bergqvist A, Thabut G, Dombret MC, Knapp D, Hamidi F, et al: Effectiveness of bronchial thermoplasty in patients with severe refractory asthma: clinical and histopathologic correlations. J Allergy Clin Immunol 2017;139:1176-1185.

16 Pretolani M, Dombret MC, Thabut G, Knap D, Hamidi F, Debray MP, et al: Reduction of airway smooth muscle mass by bronchial thermoplasty in patients with severe asthma. Am J Respir Crit Care Med 2014;190:14521454.

17 Salem IH, Boulet LP, Biardel S, Lampron N, Martel S, Laviolette M, et al: Long-term effects of bronchial thermoplasty on airway smooth muscle and reticular basement membrane thickness in severe asthma. Ann Am Thorac Soc 2016;13:1426-1428.

18 Watchorn DC, Sahadevan A, Egan JJ, Lane SJ: The efficacy of bronchial thermoplasty for severe persistent asthma: the first national experience. Ir Med J 2016;109:406.

19 Bel EH, Sousa A, Fleming L, Bush A, Chung KF, Versnel J, et al: Diagnosis and definition of severe refractory asthma: an international consensus statement from the Innovative Medicine Initiative (IMI). Thorax 2011;66: 910-917.

20 Chung KF, Wenzel SE, Brozek JL, Bush A, Castro M, Sterk PJ, et al: International ERS/ ATS guidelines on definition, evaluation and treatment of severe asthma. Eur Respir J 2014; 43:343-373.

21 Antonicelli L, Bucca C, Neri M, De Benedetto F, Sabbatani P, Bonifazi F, et al: Asthma severity and medical resource utilisation. Eur Respir J 2004;23:723-729.

22 Busse WW, Banks-Schlegel S, Wenzel SE: Pathophysiology of severe asthma. J Allergy Clin Immunol 2000;106:1033-1042.

23 Fuhlbrigge AL, Adams RJ, Guilbert TW, Grant E, Lozano P, Janson SL, et al: The burden of asthma in the United States: level and distribution are dependent on interpretation of the National Asthma Education and Prevention Program guidelines. Am J Respir Crit Care Med 2002;166:1044-1049.

24 Hekking PP, Wener RR, Amelink M, Zwinderman AH, Bouvy ML, Bel EH: The prevalence of severe refractory asthma. J Allergy Clin Immunol 2015;135:896-902.

25 Chung KF: Clinical management of severe therapy-resistant asthma. Expert Rev Respir Med 2017;11:395-402.
26 Trivedi A, Pavord ID, Castro M: Bronchial thermoplasty and biological therapy as targeted treatments for severe uncontrolled asthma. Lancet Respir Med 2016;4:585-592.

27 Benayoun L, Druilhe A, Dombret MC, Aubier M, Pretolani M: Airway structural alterations selectively associated with severe asthma. Am J Respir Crit Care Med 2003;167:1360-1368.

28 d'Hooghe JN, Eberl S, Annema JT, Bonta PI: Propofol and remifentanil sedation for bronchial thermoplasty: a prospective cohort trial. Respiration 2017;93:58-64.

29 Lee JA, Rowen DW, Rose DD: Bronchial thermoplasty: a novel treatment for severe asthma requiring monitored anesthesia care. AANA J 2011;79:480-483.

30 Mayse ML, Laviolette M, Rubin AS, Lampron N, Simoff M, Duhamel D, et al: Clinical pearls for bronchial thermoplasty. J Bronchology Interv Pulmonol 2007;14:115-123.

31 Debray MP, Dombret MC, Pretolani M, Thabut G, Alavoine L, Brillet PY, et al: Early computed tomography modifications following bronchial thermoplasty in patients with severe asthma. Eur Respir J 2017;49:1601565.

32 Debray MP, Dombret MC, Pretolani M, Thabut G, Alavoine L, Brillet PY, et al: Radiological abnormalities following bronchial thermoplasty: is the pathophysiology understood? Eur Respir J 2017;50:1702067.

33 d'Hooghe JNS, Bonta PI, van den Berk IAH, Annema JT: Radiological abnormalities following bronchial thermoplasty: is the pathophysiology understood? Eur Respir J 2017;50: 1701537.

34 d'Hooghe JNS, van den Berk IAH, Annema JT, Bonta PI: Acute radiological abnormalities after bronchial thermoplasty: a prospective cohort trial. Respiration 2017;94:258262

35 d'Hooghe JNS, Goorsenberg AWM, de Bruin DM, Roelofs J, Annema JT, Bonta PI: Optical coherence tomography for identification and quantification of human airway wall layers. PLoS One 2017; 12:e0184145.

36 Adams DC, Hariri LP, Miller AJ, Wang Y, Cho JL, Villiger $M$, et al: Birefringence microscopy platform for assessing airway smooth muscle structure and function in vivo. Sci Transl Med 2016;8:359ra131.

37 Nguyen DV, Murin S: Bronchial artery pseudoaneurysm with major hemorrhage after bronchial thermoplasty. Chest 2016;149:e95e97.

38 Balu A, Ryan D, Niven R: Lung abscess as a complication of bronchial thermoplasty. J Asthma 2015;52:740-742.

39 Funatsu A, Kobayashi K, Iikura M, Ishii S, Izumi S, Sugiyama H: A case of pulmonary cyst and pneumothorax after bronchial thermoplasty. Respirol Case Rep 2018; 6:e0286 